\title{
Automatic segmentation of the lumen of the carotid artery in ultrasound B-mode images
}

\author{
André M.F. Santos ${ }^{1(*)}$, João Manuel R.S. Tavares ${ }^{1}$, Luísa Sousa ${ }^{1}$, Rosa Santos ${ }^{2}$, Pedro \\ Castro $^{2}$ and Elsa Azevedo \\ ${ }^{1}$ Faculdade de Engenharia, Universidade do Porto, Portugal \\ ${ }^{2}$ Faculdade de Medicina, Universidade do Porto, Portugal \\ Emails:andremfs52@gmail.com, tavares@fe.up.pt, lcsousa@fe.up.pt, \\ elsaazevedo@netcabo.pt
}

\begin{abstract}
A new algorithm is proposed for the segmentation of the lumen and bifurcation boundaries of the carotid artery in B-mode ultrasound images. It uses the hipoechogenic characteristics of the lumen for the identification of the carotid boundaries and the echogenic characteristics for the identification of the bifurcation boundaries. The image to be segmented is processed with the application of an anisotropic diffusion filter for speckle removal and morphologic operators are employed in the detection of the artery. The obtained information is then used in the definition of two initial contours, one corresponding to the lumen and the other to the bifurcation boundaries, for the posterior application of the Chan-vese level set segmentation model.

A set of longitudinal B-mode images of the common carotid artery (CCA) was acquired with a GE Healthcare Vivid-e ultrasound system (GE Healthcare, United Kingdom). All the acquired images include a part of the CCA and of the bifurcation that separates the CCA into the internal and external carotid arteries. In order to achieve the uppermost robustness in the imaging acquisition process, i.e., images with high contrast and low speckle noise, the scanner was adjusted differently for each acquisition and according to the medical exam.
\end{abstract}

The obtained results prove that we were able to successfully apply a carotid segmentation technique based on cervical ultrasonography. The main advantage of the new segmentation method relies on the automatic identification of the carotid lumen, overcoming the limitations of the traditional methods.

Keywords: Medical imaging; common carotid artery; internal and external carotid arteries; ultrasound imaging; image segmentation; Chan-Vese model.

\section{INTRODUCTION}

The common carotid artery (CCA) is the one that supplies the human head, specifically the front part of the brain, and neck, with oxygenated blood. Like other arteries, which purpose relies in the supply of blood from the heart, as the coronary arteries, the carotid is also in risk of developing several diseases, like atherosclerosis, known as the "hardening of the artery", after the accumulation of fatty substances, i.e. lipoproteins, in the artery walls. This accumulation is known as "plaque" and decreases the blood supply. The carotid artery, specifically at the bifurcation, which separates the external (ECA) and internal (ICA) carotid arteries, is one very susceptible to atherosclerosis, mainly because of the high hemodynamic forces that can be found at the bifurcation and branching structures.

Non-invasive ultrasound imaging has been widely used in the diagnosis of cardiovascular diseases, in particular concerning the atherosclerosis with the evaluation of the intima-media thickness (IMT), assessing the distance between the lumen of the carotid artery, that is, where the blood flows, and the inner boundary of the adventitia. This measure, and consequent diagnosis of atherosclerosis among other cardiovascular diseases, is performed with the aid of B-mode ultrasound imaging, requiring the detection of not only the lumen boundaries but as well as of both the near and far adventitia. Therefore, it has been and continues to be a great interest in the efficient automatic segmentation of the adventitia and lumen boundaries in B-mode ultrasound images of the carotid artery. According to Halenka (1999), in this type of images the carotid adventitia appears as two almost parallel lines, known due to their echogenic characteristics, separated in the middle by a hipoechogenic space, known as the "double line" pattern (Halenka, 1999). 
Ultrasound B-mode imaging has been the most widely used technique in image-based cardiovascular diagnosis due to the fact of the carotid being a superficial artery and quite suitable for this type of imaging. However, B-mode images present difficulties, specifically in the segmentation of the structures involved, due to several imaging characteristics like low contrast, speckle noise, echo shadows and artifacts, which lead to images of very poor quality that usually require the interaction of an expert. Some works that use several statistical distributions can be found in the literature to cope the granular speckle noise in non-compressed ultrasound signals, like, for example, the Rayleigh distribution (Wagner et al., 1983; Sarti et al., 2004) and Kdistribution (V. Dutt et al., 1994; R.C. Molthen et al., 1993). However, most of the signals that are actually used in ultrasound imaging and medical practice are log-compressed signals, which are therefore, unsuitable for the application of statistical distributions because of the reduced intensity range that is characteristic of this type of signal. In 2006, Noble (Noble et al., 2006) described the success of texture segmentation techniques in the classification of breast masses and liver and kidney tissues in ultrasound images. However, the segmentation of the carotid artery tends to be more difficult due to the extremely low degree of discrimination of this structure in the usual ultrasound B-mode images.

Ultrasound imaging represents an extreme and complex challenge to the automatic segmentation algorithms, as for the reasons described earlier, as for the amount of boundary edges that may be missing in the image, leading to gaps in the vessel boundaries. Additionally, as different anatomical regions of the carotid can be acquired in ultrasound B-mode imaging, and also due to the variability of its shape among subjects, a modelbased segmentation is not appropriate. Despite these difficulties, there has been an increasing interest in ultrasound imaging-based medical diagnosis as consequence of the technological advances verified in this methodology, not only in terms of image quality, but also because of its non-invasive characteristics and low cost (Rui Rocha et al., 2011).

The desired segmentation can be addressed by two main steps: i) the definition or estimation of a region of interest (ROI) of the carotid artery in the B-mode ultrasound image and ii) the delineation of the boundaries of the structure desired, which depends on the ROI defined and can be the artery lumen, intima or adventitia. For this reason, we may consider that the two steps are not independent from each other, since the correct delineation of the artery wall in the segmentation algorithm is strictly connected to the right definition of the ROI.

In this paper, a method is proposed for the automatic identification of the lumen region and consequent segmentation of the lumen boundaries in longitudinal B-mode images of the CCA. The method searches for hipoechogenic structures in the input image, and the lumen region of the CCA is identified based on mean and standard deviation calculations concerning the image intensity. Afterwards, the lumen and bifurcation boundaries of the carotid artery are identified through the application of a geometrical model, in particular, using the Chan-Vese level set model. The method is robust to speckle noise, does not require human interaction and can adjust well the segmentation contours to the lumen boundaries represented in the input images.

\section{METHODS}

The approach developed is depicted in Figure 1. It starts by detaching the ultrasound data to be analyzed from other features like menus, patient data, etc., followed by the definition of two 2DH (2D histograms) representing for each image pixel the mean and standard deviation values of the intensity within a neighborhood. With the application of a Gaussian low-pass filter, for speckle noise reduction, the combining of the smoothed image with the two 2D histograms allows the identification of the lumen region of the carotid artery based on its hipoechogenic characteristics. The result is an estimation of the lumen and bifurcation boundaries represented in the input image. 


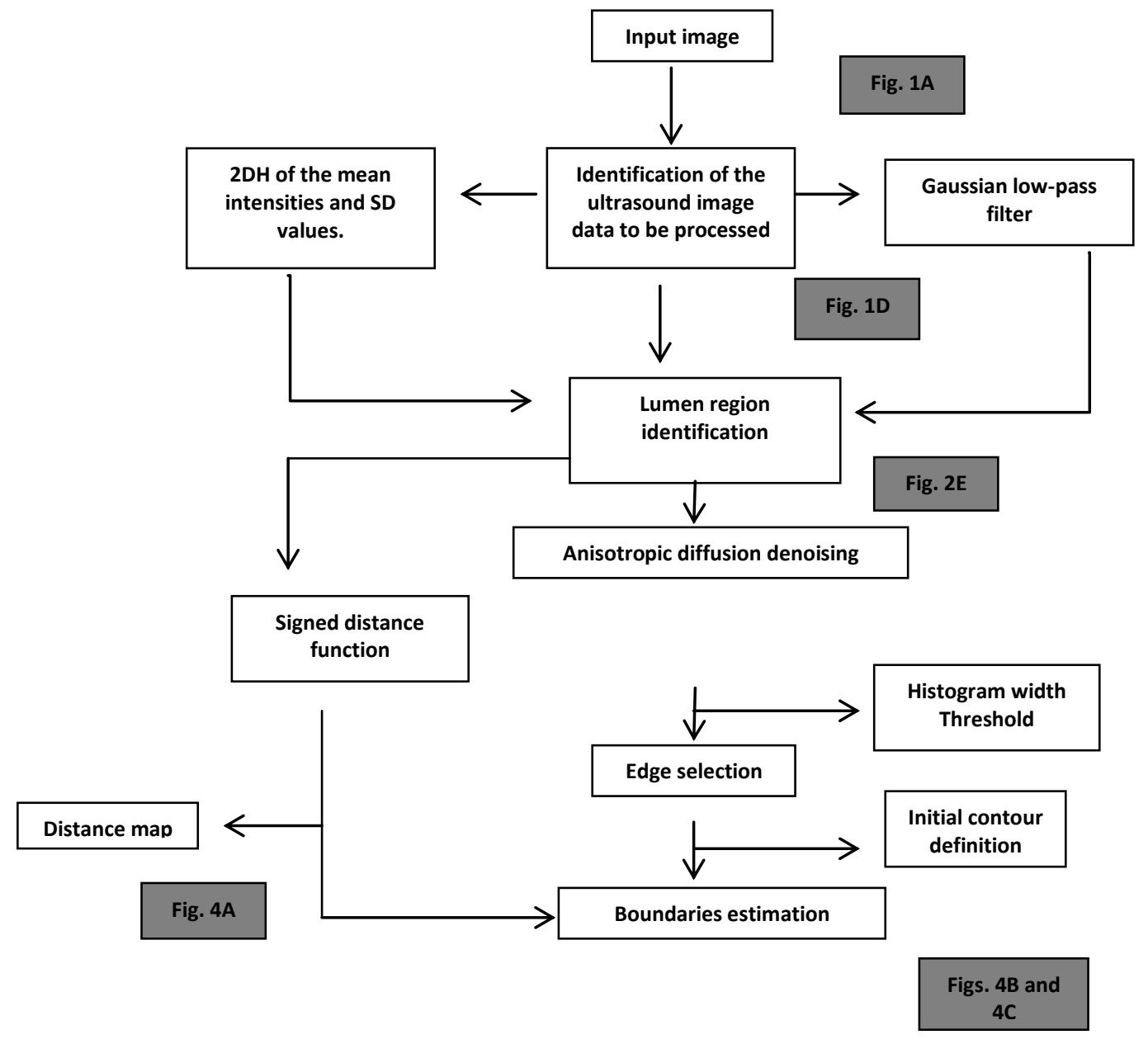

Figure 1: Main steps of the method developed.

Data set: A set of 11 longitudinal B-mode images of the CCA was acquired in 256 gray levels using a GE Healthcare Vivid-e ultrasound system (GE Healthcare, United Kingdom). All the images include part of the CCA and the bifurcation that separates the CCA into the ICA and ECA. In order to achieve high robustness in the acquisitions, i.e., images of high contrast and low speckle noise, the parameters of the scanner were defined according to the characteristics of each medical exam under evaluation.

Identification of the image area and consequent reduction of the image size: As a first step, we intend to reduce the image area, eliminating any possibility of detecting unwanted features that do not belong to the ultrasound data to be analyzed. This procedure also reduces the time required in the posterior steps of image processing and segmentation. The reduction of the image data consists in the definition of a rectangular area involving the carotid artery (Golemati et al., 2007). With this goal in mind, four points are identified by the following procedure: (1) Morphological opening of the original image, using a circular element to remove unwanted objects such as characters; (2) Image binarization, as such, the areas outside the region of the ultrasound data to be analyzed are discarded; (3) Finally, the four points that correspond to the first and last nonzero lines and columns of the binary image are identified. These points are the vertices of the rectangular area in which all the further tasks of image processing and segmentation are performed.

Lumen region identification: This procedure is based on the study performed by Liboni et al. (2007) to develop a computer-based tracing of the carotid artery. According to these authors, the carotid characteristics in an ultrasound image can be addressed using a model of variable intensity distribution over the carotid regions. It is precisely this idea that is used here for the automatic identification of the lumen of the carotid artery. 
Pixels belonging to the lumen region of the carotid artery are those characterized by both low mean and standard deviation intensity values (Liboni et al. (2007)). In order to proceed with this identification, 2D histograms are built: For each pixel of the image to be analyzed, it is calculated within a neighborhood the mean and standard deviation intensity values; both values are then normalized and grouped into a set of classes.

A row-wise intensity distribution is built for each column of the ultrasound image region to be analyzed so the pixels corresponding to the carotid artery can be identified. However, the image data must be previously processed for speckle noise removal and attenuating the high intensity noisy points in the intensity distribution; in this task, a Gaussian low-pass filter is used. As mentioned previously, pixels belonging to the lumen region of the carotid artery are those characterized by their low mean and standard deviation intensity values. Having this into consideration, those pixels are identified in the intensity distributions built as being those related to the minimum values presented, which are frequently between the local maximums corresponding to the near and far adventitia layers, or corresponding to the walls of the ICA and ECA, or in the interval between these two borders, if it is considered a column of the image containing pixels belonging to the carotid bifurcation. Based on Liboni et al. (2007) approach, the identification process starts from the bottom of the image to be analyzed, i.e. from the highest row index, and moves upwards along the rows in order to correctly identify the first pixel of the first maxima which as possibly corresponds to the far adventitia of the carotid that is usually associated to the brightest structure of the ultrasound image of the carotid artery. Having this first pixel estimated as possibly belonging to the far adventitia, the method continues the lumen identification moving upwards and searching for a pixel possible belonging to the lumen region. Taking into account the row of the pixel that corresponds to the far adventitia, the pixel possibly belonging to the lumen is the first minima point after the far adventitia pixel. Also, its neighborhood mean and standard deviation intensity values must match the chosen criteria for the $2 \mathrm{D}$ histogram.

Lumen edges identification: Having obtained the correct identification of a group of pixels belonging to the lumen of the carotid artery presented in the input image, the definition of a suitable mask for a level set-based segmentation is possible. However, some processing techniques must be applied to the image to be segmented in order to assure the robustness of the segmentation process. Hence, an anisotropic diffusion filter is applied in order to attenuate the high amount of speckle noise that is commonly present; the filter proposed in (Perona and Malik, 1990) with a 2D network structure of 8 neighboring nodes for diffusion conduction was chosen to accomplish such smoothing. Then, it is applied a morphological closing operator in order to merge small "channels" and "openings". Thirdly, a threshold based on the value defined based on the image histogram is performed. This threshold results in a binary image on which is applied the Sobel gradient operator in order to identify the edge points. The binary image obtained with the application of the Sobel operator combined with the information relevant to the pixels that belong to the lumen region of the carotid allow the identification of the edges correspondent to the superior and inferior wall of the lumen of the carotid artery. Figure $2 \mathrm{E}$ shows that combination; in this figure, it can be observed the pixels of the lumen candidate string with the highest column value (located in the rightest part of the image), which can be searched in the binary image resulted from the Sobel operator, the pixels above and below (at the same column) with value 1 (one). Having the row and column of these two pixels found, one belonging to the superior lumen edge and the other to the inferior one, it is possible to trace the remainder of the edges in the Sobel binary image and store the coordinates of each pixel in a string. As such, two strings are built having the coordinates of each pixel belonging to the superior and inferior lumen edges. Posteriorly, a new string is defined containing the pixels belonging to the bifurcation edge in the binary image, knowing that the bifurcation is located between the superior and inferior walls of the carotid. Finally, two masks are defined for posterior application of the geometrical model of Chan-Vese for the segmentation of both bifurcation and common carotid artery walls, as shown in Figure 4. These masks are created with the information of the superior and inferior walls of the carotid artery, as well as its bifurcation, by filling its interior with pixels of value 1 (one), and the outside with pixels of value 0 (zero), creating a binary image.

Segmentation of the lumen and bifurcation boundaries of the carotid artery using the Chan-Vese geometrical model: The Chan-Vese level set is a powerful segmentation method that can be used to detect the lumen boundaries of the carotid artery using the two masks built in the previous step as initial contours. This segmentation approach is well known for its high flexibility and accuracy as is a region-based model independent of gradient information. This independence makes the segmentation robust to cases in which 
gaps exist in the boundaries of the carotid as usually occur. The segmentation both of the lumen and bifurcation boundaries of the carotid artery is based on the work developed by Lankton and Tannenbaum (2008) that defined a local-based framework according to the active contour moves according to an internal energy defined in the Chan-Vese approach using a constant intensity model. The framework starts with the input of an initial contour, here, one of the masks built in the previous step, and the definition of a signed distance function $\phi$ defined as:

$\phi=E_{d}(m)-E_{d}(1-m)+\left(m-\left(\frac{1}{2}\right)\right)$

where $m$ represents the initial contour and $E_{d}$ is the Euclidean distance transform of the considered binary image, assigning for each pixel, the distance between them and the nearest nonzero value.

Let $C$ represent a closed contour, as the zero level of $\phi$, i.e., $C=\{x \mid \phi(x)=0\}$, where its interior is expressed respectively as:

$$
H \phi(x)=\left\{\begin{array}{ll}
1 & , \phi(x)<-\epsilon \\
0 & , \phi(x)>\epsilon \\
\frac{1}{2}\left\{1+\frac{\phi}{\epsilon}+\frac{1}{\pi} \sin \left(\frac{\pi \phi(x)}{\epsilon}\right)\right\} & , \text { otherwise }
\end{array},\right.
$$

Similarly, the exterior of $\mathrm{C}$ is defined by: $\phi(x)=[1-H \phi(x)]$.

Having $x$ and $y$ as independent variables representing the coordinates of a pixel in the domain $\Omega$ of an image, the following equation represents a function defining a region of interest (ROI) of radius $r$, with value 1 (one) inside and 0 (zero) outside:

$$
B(x, y)=\left\{\begin{array}{l}
1,\|x-y\|<r \\
0, \quad \text { otherwise }
\end{array}\right.
$$

With eq. (4), the energy functional can be defined as:

$$
E(\phi)=\int_{\Omega_{x}} \delta \phi(x) \int_{\Omega_{y}} B(x, y) \cdot F(I(y), \phi(y)) d y d x,
$$

where $\delta \phi(x)$ prevents the development of new contours by ensuring that $C$ does not undergo sudden changes in its geometry. On the other hand, it will allow certain parts of the contour $C$ to separate or combine within each other. Each pixel in this term is masked to $B(x, y)$ ensuring that only the local information surrounding $C$ will be used.

The smoothness of the contour $C$ is assured through the application of a regularization term that penalizes its arc length. The weight of this penalty is controlled by the parameter $\lambda$ in the new equation of the energy functional:

$$
E(\phi)=\int_{\Omega_{x}} \delta \phi(x) \int_{\Omega_{y}} B(x, y) \cdot F(I(y), \phi(y)) d y d x+\lambda \int_{\Omega_{x}} \delta \phi(x)\|\nabla \phi(x)\| d x
$$

Lanktom and Tannenbaum (Lanktom and Tannenbaum, 2008) proposed the introduction of specific energies into the generic framework described previously, including the Chan-Vese energy that is expressed as:

$$
E_{C V}=\int_{\Omega_{y}} H \phi(y)(I(y)-u)^{2}+(1-H \phi(y))(I(y)-v)^{2} d y
$$

where $u$ and $v$ are global mean intensities of the interior and exterior regions of $C$ given as:

$$
u=\frac{\int_{\Omega_{y}} H \phi(y) \cdot I(y) d y}{\int_{\Omega_{y}} H \phi(y) d y},
$$




$$
v=\frac{\int_{\Omega y}(1-H \phi(y)) \cdot I(y) d y}{\int_{\Omega y}(1-H \phi(y)) d y} .
$$

The corresponding Chan-Vese internal energy function is based on the local mean intensities $u_{x}$ and $v_{x}$, instead of $u$ and $v$ :

$$
\begin{aligned}
& F_{C V}=H \phi(y)\left(I(y)-u_{x}\right)^{2}+(1-H \phi(y))\left(I(y)-v_{x}\right)^{2}, \\
& u_{x}=\frac{\int_{\Omega_{y}} B(x, y) H \phi(y) \cdot I(y) d y}{\int_{\Omega_{y}} B(x, y) H \phi(y) d y}, \\
& v_{x}=\frac{\int_{\Omega_{y}} B(x, y)(1-H \phi(y)) \cdot I(y) d y}{\int_{\Omega_{y}} B(x, y)(1-H \phi(y)) d y} .
\end{aligned}
$$

Eq. (10) can now be replaced in the energy functional of the framework given by eq. (6) defining a localized energy. However, to obtain the curvature flow regularization term, eq. (10) must first be derivated:

$$
\begin{aligned}
& \nabla_{\phi(y)} F=\delta \phi(y)\left(\left(I(y)-u_{x}\right)^{2}-\left(I(y)-v_{x}\right)^{2}\right), \\
& \frac{\partial \phi}{\partial t}(x)=\delta \phi(x) \int_{\Omega_{y}} B(x, y) \nabla_{\phi(y)} F(I(y), \phi(y)) d y+\lambda \delta \phi(x) \operatorname{div}\left(\frac{\nabla \phi(x)}{|\nabla \phi(x)|}\right) \Leftrightarrow \\
& \Leftrightarrow \frac{\partial \phi}{\partial t}(x)=\delta \phi(x) \int_{\Omega_{y}} B(x, y) \delta \phi(y)\left(\left(I(y)-u_{x}\right)^{2}-\left(I(y)-v_{x}\right)^{2}\right) d y+\lambda \delta \phi(x) \operatorname{div}\left(\frac{\nabla \phi(x)}{|\nabla \phi(x)|}\right) .
\end{aligned}
$$

The Chan-Vese energy function finds its minimum when the interior and exterior of the curve $C$ are closer to the global mean intensities $u$ and $v$, while in the localized version, the minimum is obtained when they are closer to the local mean intensities $u_{x}$ and $v_{x}$.

For the segmentation of the bifurcation boundaries, the mask illustrated in Figure $3 \mathrm{~g}$ is chosen as the initial contour. The level set for the segmentation of these boundaries must be flexible in order to reach the limit of the bifurcation walls. On the other hand, for the segmentation of the lumen boundaries, the mask illustrated in Figure $3 \mathrm{f}$ is defined and the development contour $C$ has to be properly controlled and somehow attenuated in order to prevent its development towards other structures near the carotid artery. The circular ROI $B(x, y)$ has also to be chosen narrow in order to prevent larger intensity variations during the contour $C$ development along the Chan-Vese energy minimization process.

Contour smoothness: Finally, the obtained contours must be smoothed: Firstly, through a cubic spline interpolation and secondly, by projecting all the resulting points of the contour towards a local regression line. Once again, a ROI is defined for each point of the contour, regarding the number of points in the neighborhood that contribute for the computation of the local regression line. In this process, for each pixel we defined an 8-connected-neighborhood for its computation.

\section{RESULTS}

The parameters of the method proposed used were defined through several experimental tests. Figure 2, shows an example of the first step of the method that aims to identify automatically in an input B-mode ultrasound image the rectangular area enclosing the ultrasound data to be further analyzed.

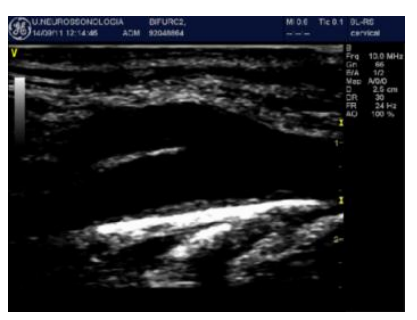

(a)

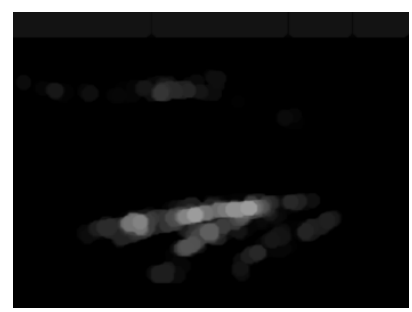

(b) 


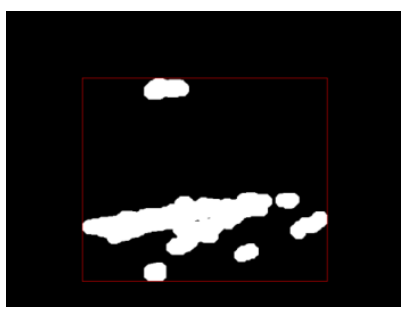

(c)

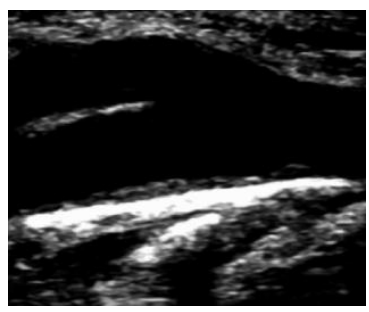

(d)

Figure 2: Automatic identification and consequent detaching of the ultrasound data to be further analysed: (a) Original image; (b) Image resultant of the morphological opening; (c) Resultant thresholded image overlapped with the rectangular area found to crop the original image defined based on the first and last nonzero pixels in the lines and columns; (d) Resultant image with the desired ultrasound image data.

Figure 3 demonstrates an example of the identification of the lumen region of the CCA in an ultrasound image of the carotid artery achieved by our method. Figure 3 a shows the cropped image after the application of the Gaussian low-pass filter with a kernel size of $40 \times 40$ pixels and $\sigma=10$; all the possible pixel candidates for the lumen region of the CCA are represented with value 1's (corresponding to the white color) in the binary image presented in Figure $3 \mathrm{~b}$. Finally, the pixels that were identified as belonging to the lumen region of the CCA and as being closer to the lumen boundaries of the CCA are posteriorly used in the definition of the initial contour for the application of Chan-Vese segmentation model.

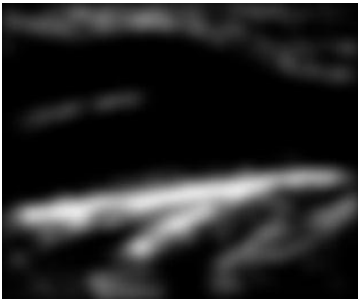

(a)

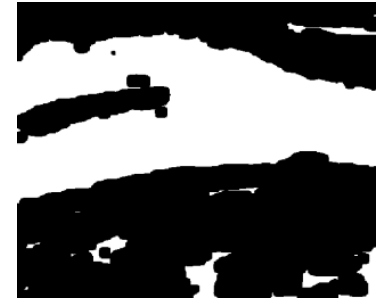

(b)

Figure 3: Automatic identification of the lumen region of the CCA in one B-mode ultrasound image of the carotid artery: (a) Cropped image after the application of a Gaussian low-pass filter; (b) All possible pixel candidates for the lumen region of the CCA (in white).

Figure 4 demonstrates the described steps of our method for the identification of the lumen edges: Figure 4a illustrates the cropped image in grayscale, i.e., with pixel values varying from 0 (zero) to 255 , with 0 (zero) corresponding to the black color and 255 to the white color, while Figure $4 \mathrm{~b}$ illustrates the same image after the application of the anisotropic filter proposed by Perona and Malik (1990) for speckle noise removal. After the application of a threshold with a value correspondent to the first $15 \%$ of the image histogram width, the image illustrated in Figure $4 \mathrm{c}$ is obtained. In Figure 4d, the pixels identified in the binary image resultant of the Sobel edge detector as belonging to the superior and inferior walls of the carotid artery and to its bifurcation are represented in green. Finally, Figures $4 \mathrm{e}$ and $4 \mathrm{f}$ illustrate the two masks used to define the initial contours for the posterior Chan-Vese level set-based segmentation.

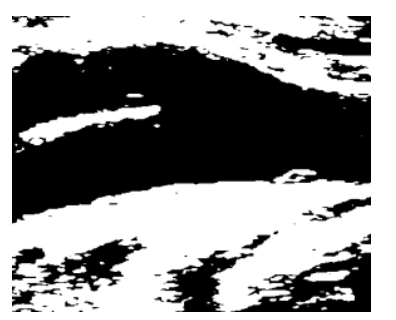

(a)

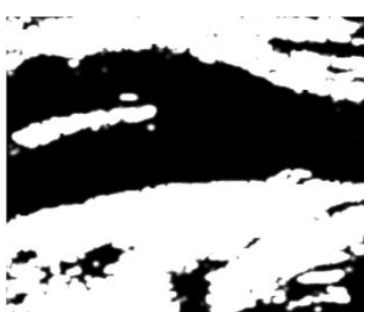

(b)

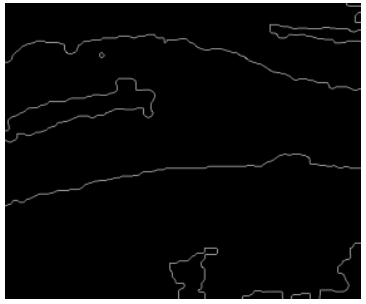

(c) 


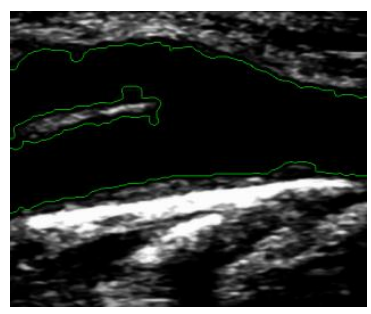

(d)

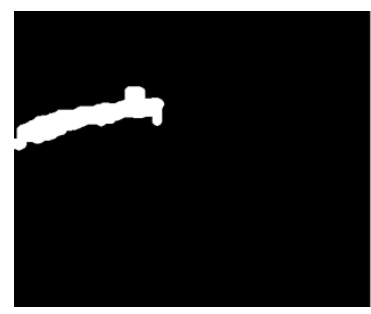

(e)

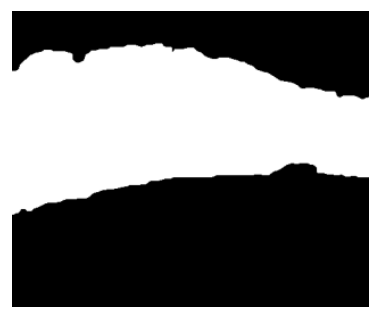

(f)

Figure 4: Lumen edges identification: (a) Cropped image in grayscale; (b) Resultant image after the application of an anisotropic diffusion filter for speckle noise removal; (c) Image after the application of the Sobel edge detector; (e) Original cropped image overlapped by the pixels identified in image (d) as belonging to the superior and inferior walls of the carotid artery represented in green; (e and f) The two binary images defining the masks to be used in the level set-based segmentation.

Figure 5 demonstrates the results of the application of the geometric level set model based on the Chan-Vese energy minimization developed by Lanktom and Tannenbaum (Lanktom and Tannenbaum, 2008), in the image shown in Figure $4 \mathrm{a}$ and starting with the initial contours defined by the masks visible in Figures $4 \mathrm{e}$ and 4f. Thus, Figure 5a shows the segmentation obtained for the bifurcation of the carotid artery boundaries, while Figure $5 \mathrm{~b}$ reveals the segmentation obtained by our method for the lumen boundaries.

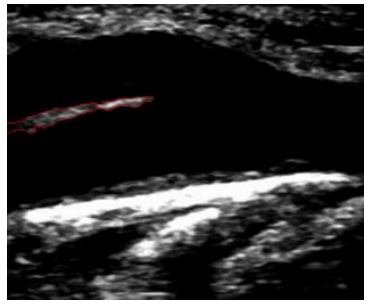

(a)

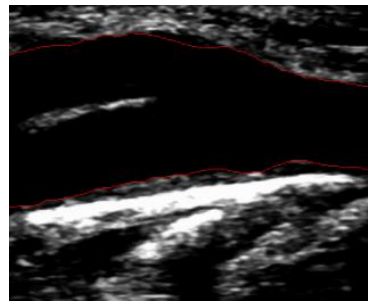

(b)

Figure 5: Segmentation using the geometric model level set based on the Chan-Vese energy minimization: (a) segmentation obtained for the bifurcation boundaries of the carotid artery; (b) segmentation obtained for the lumen boundaries of the carotid artery.

The smoothed contours obtained for the lumen and bifurcation boundaries are both represented in the cropped B-mode ultrasound image. For exemplification purpose, Figure 6 shows the smoothed contours obtained by our method in 6 of the 11 B-mode ultrasound images tested. From a visual based analysis of the images included in this figure, one can conclude that the segmentation results of our method are very good.

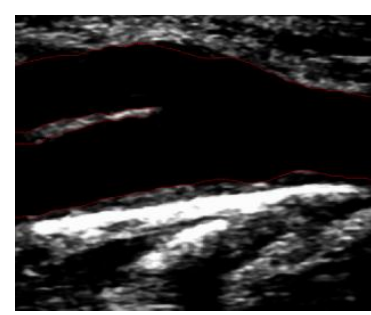

(a)

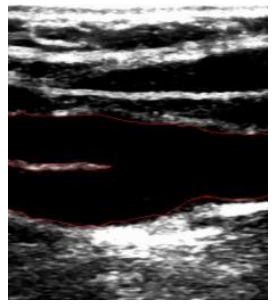

(b)

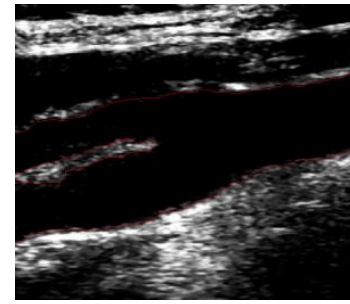

(c) 


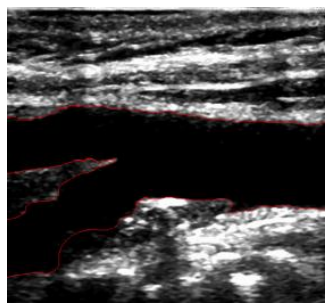

(d)

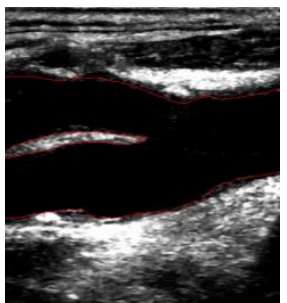

(e)

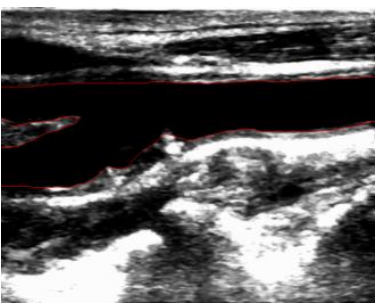

(f)

Figure 6: Examples of the segmentation of the lumen and bifurcation boundaries of the carotid artery achieved by our method in $6 \mathrm{~B}$-mode ultrasound images.

For a quality assessment of the segmentation results obtained by our method, the same $11 \mathrm{~B}$-mode ultrasound images were manually segmented by a specialist on this medical exam. The manual segmentation of each image consisted in the definition of a set of border points on the lumen and bifurcation of the carotid artery. From the points defined, the correspondent contour of the carotid artery was defined by a cubic interpolation and then smoothed. Afterwards, the evaluation of the our computational proposed for the automatic segmentation of the carotid artery was performed using two different procedures: one focus on area-based metrics, by the comparison of the area enclosed between the contour obtained by the computational method with the one obtained by the manual segmentation, Figure 8; and the second one focus on a distance-based metric of the maximum minimal distance of the points that were defined by the specialist with the closest one from the contour obtained by the computational method, through the definition of the normal line that intersects the correspondent point of the contour manually drawn.

The area-based metric procedure was based on the approach proposed in (Yi Guo et al., 2011), according to which the evaluation is achieved by the pixel-analysis of true positives $(T P)$, false positives $(F P)$, true negatives $(T N)$ and false negatives $(F N)$, and the parameter related to the area overlap $(A O)$ by the two contours, which is calculated as:

$$
A O=\frac{T P}{T P+F P+F N} * 100 .
$$

The values found for this parameter regarding the images under study are shown in Table 1. From the values present, one can conclude that the values of $\mathrm{AO}$ vary between $94.64 \%$ and $98.89 \%$, being the mean area equal to $96.78 \%$.

Concerning the distance-based metric, it is firstly found how many points are coincident on the two contours: the one resultant by the automatic segmentation and the correspondent one manually defined. Then, for each non-coincident point of the contour manually defined, it is defined a straight line normal to the contour and passing through that point. The definition of this line facilitates the identification of the correspondent point in the related contour obtained by our automatic method, and the consequent calculation of the distance between the correspondent points, i.e., the distance error $\left(e_{i}\right)$. As such, the maximum and mean errors can be found, Figure 7.

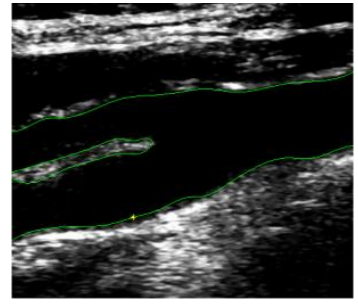

(a)

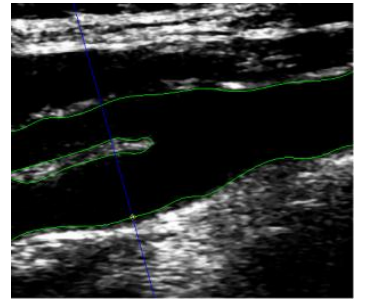

(b) 


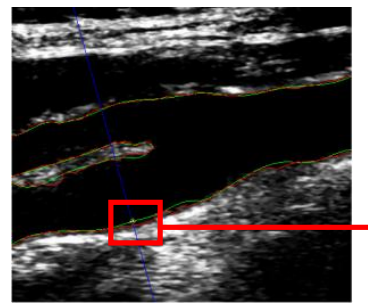

(c)

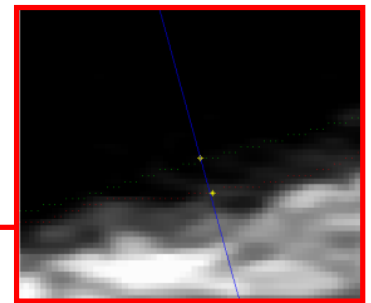

(d)

Figure 7: Example of a non-coincident point and calculation of the associated distance: (a) Contour manually defined draw in green and the non-coincident point in yellow; (b) Definition of the straight line normal to the contour manually defined and passing through the non-coincident point; (c) Difference between the contours manually and automatically obtained for the non-coincident point; (d) Zoom of the rectangular area identified in red in image (c).

Figure 8 shows the contours obtained by an expert and our segmentation method; the values calculated for the quality evaluation of the results are indicated in Table 1.

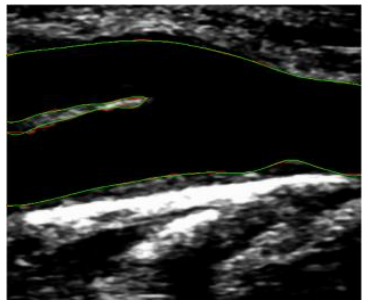

(a)

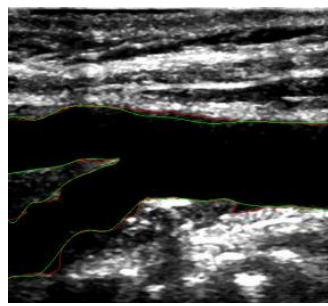

(d)

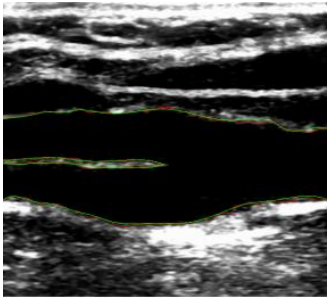

(b)

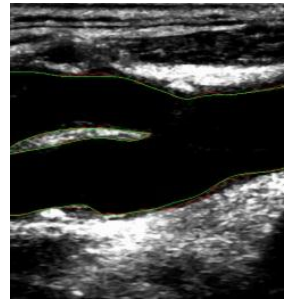

(e)

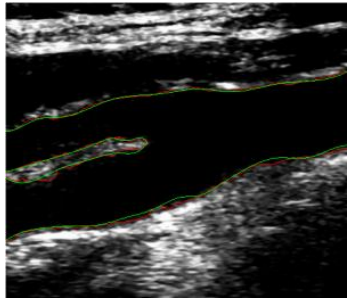

(c)

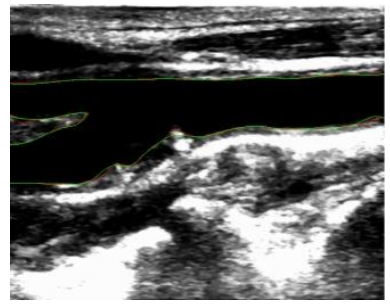

(f)

Figure 8: Contours obtained by an expert (in green) and by our segmentation method (in red) in 6 B-mode ultrasound images.

Table 1: Comparison between the results of the automatic and manual segmentations for the $11 \mathrm{~B}$-mode ultrasound images 6 of which are shown in Figure 8.

\begin{tabular}{|c|c|c|c|c|c|c|c|}
\hline Images & $\begin{array}{c}\text { Size } \\
\text { (Rows x } \\
\text { Columns) }\end{array}$ & $\begin{array}{c}\text { Number of } \\
\text { points } \\
\text { manually } \\
\text { defined }\end{array}$ & $\begin{array}{c}\text { Number of } \\
\text { coincident } \\
\text { points }\end{array}$ & $\begin{array}{c}\text { Number of } \\
\text { non- } \\
\text { coincident } \\
\text { points }\end{array}$ & $\begin{array}{c}\text { Maximum } \\
\text { error (pixels) }\end{array}$ & $\begin{array}{c}\text { Mean } \\
\text { error } \\
\text { (pixels) }\end{array}$ & $\begin{array}{c}\text { Area } \\
\text { Overlapped } \\
\text { (\%) }\end{array}$ \\
\hline \#1 (Fig. 8a) & $409 \times 504$ & 46 & 35 & 11 & 1 & 0.239 & 98.89 \\
\hline$\# 2$ (Fig. 8b) & $490 \times 533$ & 73 & 42 & 21 & 1.414 & 0.293 & 97.13 \\
\hline$\# 3$ (Fig. 8c) & $417 \times 482$ & 66 & 44 & 22 & 8 & 0.66 & 95.92 \\
\hline \#4 & $490 \times 522$ & 45 & 34 & 11 & 1 & 0.244 & 96.88 \\
\hline$\# 5$ (Fig. 8d) & $486 \times 525$ & 56 & 34 & 22 & 8.246 & 0.978 & 95.42 \\
\hline
\end{tabular}




\begin{tabular}{|c|c|c|c|c|c|c|c|}
\hline$\# 6$ & $493 \times 351$ & 39 & 29 & 10 & 8 & 0.833 & 96.67 \\
\hline$\# 7$ & $499 \times 457$ & 38 & 24 & 14 & 9.84 & 0.47 & 94.64 \\
\hline$\# 8$ (Fig. 8e) & $496 \times 457$ & 44 & 33 & 11 & 7.615 & 0.635 & 97.73 \\
\hline$\# 9$ (Fig. 8f) & $499 \times 631$ & 43 & 33 & 10 & 5.385 & 1.419 & 97.76 \\
\hline$\# 10$ & $473 \times 505$ & 57 & 45 & 12 & 3.223 & 0.33 & 98.03 \\
\hline$\# 11$ & $490 \times 457$ & 60 & 50 & 10 & 2.712 & 0.27 & 97.91 \\
\hline
\end{tabular}

\section{DISCUSSION}

The proposed method provides the fully automatic segmentation of the arterial lumen and bifurcation boundaries of the carotid artery in longitudinal ultrasound images. Its main advantage relies on the automatic identification of the carotid lumen based on its hipoechogenic characteristics overcoming the limitations of the traditional methods. In our experimental dataset, the method has proven to be highly efficient, robust and accurate.

As can be verified in Figure 4b, the application of the anisotropic diffusion filter proposed by Perona and Malik (1990) is satisfactory, allowing the correct distinction of the carotid artery from other near vessels and small features, which greatly facilitates the posterior detection of the boundaries of the carotid for the definition of the initial contours for the application of the Chan-Vese model. In all tested images, the initial contours for the Chan-Vese model have been successfully defined, i.e., very close to the true lumen and bifurcation boundaries of the carotid artery, which is an advantage in the proposed method, since it significantly increases the robustness of the segmentation process.

Regarding the results of the proposed method illustrated in Figure 6, we can consider them to be very satisfactory. As it can be seen in the images included in this figure, there was an overall correct identification of the boundaries of the lumen and bifurcation of the carotid artery. The best segmentation was achieved in the image of Figure 6a since, although it is the case with the lumen, intima-media and adventitia boundaries that are best defined in terms of contrast, hipoechogenic characteristics and noise, the contours obtained are highly smoothed and perfectly adjusted to the boundaries of the carotid artery. The bifurcation is also very well defined in this image. The same not happened for the carotid in the image of Figure 6d; in this case, it is clear that the contrast of the bifurcation of this carotid artery is really very poor and with a high level of noise and gaps in its interior. In spite of the complexity of this image, the segmentation obtained by our method can be globally considered as satisfactory.

The images tested were manually segmented by an expert and a statistical comparison with the segmentations obtained by the automatic segmentation method was performed. The results obtained (Table 1), with a mean area overlapped of $96.78 \%$, and a maximum distance of 9.85 pixels between the non-coincident points of the contours obtained automatically and manually, confirm the good quality of the proposed segmentation method. The overall error in the distances between the non-coincident points of the contours automatically and manually obtained is very low, with the maximum value equal to 1.419 pixels for the contours of Figure $8 \mathrm{f}$, which proves that the contours resulting from the automatic segmentation are very similar to the ones manually defined by the expert.

\section{CONCLUSIONS AND FUTURE WORK}

We were able to successfully apply a carotid segmentation method based on cervical ultrasonography. The main advantage of the novel segmentation method relies on the automatic identification of the carotid lumen, overcoming the limitations of the traditional methods.

As a future work, we will test our method using more B-mode ultrasound images, including images of carotid arteries of patients with severe atherosclerosis. With the obtained contours from the segmentation of the carotid artery, 3D models will be building using algorithms of data interpolation, geometrical meshing and smoothing. With additional images acquired by computerized angiography, we expect to be able to building 
accurate 3D models for carotid arteries that can be posteriorly deformed and adjusted to the data obtained with the segmentation of the lumen and bifurcation structures of the carotid artery in ultrasound B-mode images. This will allow the achievement of truthful 3D models for carotid arteries from B-mode ultrasound images.

\section{ACKNOWLEDGMENTS}

This work was done in the scope of the projects with references PTDC/EEA-CRO/103320/2008, PTDC/SAUBEB/102547/2008, UTAustin/MAT/0009/2008 and UTAustin/CA/0047/2008, financially supported by Fundação para a Ciência e a Tecnologia (FCT) in Portugal.

\section{REFERENCES}

Abdel-Dayem, A. R., El-Sakka ,M.R., "Carotid artery image segmentation using fuzzy region growing", International Conference of Image Analysis and Recognition, 3656, 869-878, (2005).

Abdel-Dayem, A.R., El-Sakka, M.R., Carotid artery contour extraction from ultrasound images using multiresolution analysis and watershed segmentation scheme. Graphics, Vision and Image Processing. Cairo, Egypt, 5(9), 1-10 (2005).

Antiga, L., Ene-Irodache, B., Remuzzi, A., Computational geometry for patient-specific reconstruction and meshing of blood vessels from MR and CT angiography. IEEE Transactions on medical imaging. 22(5), 674684 (2005).

Barratt, D.C., Ariff, B.B., Humphries, K.N., Thim, S.A.M., Hughes, A.D., Reconstruction and quantification of the carotid artery bifurcation from 3D ultrasound images. IEEE Transaction on medical imaging, 23(5), 567-583 (2005).

Chan, K.L., "Ultrasound tissue characterization using fractal feature", IEEE conference of acoustic sensing and imaging, 369, 193-188 (1993).

Chen, Y., Huan, H.D., Tagare, H.D., Rao, M., Wilson, D., Geiser, E.A., "Using prior shape and intensity profile in medical image segmentation", IEEE international conference of computational vision, 2, 1117-1125 (2003).

Cheng, D.C., Billich, C., Liu, S.H., Qiu, Y.C., Shen, Y.L., Brambs, H.J., Trucksass, A.S., Schutz, U.H.W., "Automatic detection of the carotid artery boundary on cross-sectional MR image sequences using circle model guided dynamic programming", Biomedical Engineering Online, 10, 10-26 (2011).

Cheng, D., Schmidt-Trucksass, A., Cheng, K., Sandrock, M., Pu, Q., Burkhardt, H., "Automatic detection of the intimal and the adventitial layers of the common carotid artery wall in ultrasound B-mode images using snakes", International conference on image analysis and processing, Venice, $452-457$ (1999).

Cheng, D., Schmidt-Trucksass, A., Cheng, K., Sandrock, M., Pu, Q., Burkhardt, H., "Using snakes to detect the intimal and adventitial layers of the common carotid artery wall in sonographic images", Computer methods and programs in biomedicine, 67, 27-37 (2002).

Cheng, J., Ding, M., Zhang, X., "Geometric active contour model and its application to carotid plaque detection", International conference computation and biomedical instrumentation, Wuhan, Hubei, 101-104 (2011). 
Cheng, J., Ding, M., Zhang, X., "Geometric active contour model and its application to carotid plaque detection", International conference computation and biomedical instrumentation, Wuhan, Hubei, 101-104 (2011).

Chiu, B., Beletsky, V., Spence, J.D., Parrag, G., Fenster, A., "Analysis of carotid surface morphology using three-dimensional ultrasound imaging", Physics in Medicine and Biology., 54, 1149-1167 (2009).

Cremers, D., Kohlberger, T., Shcorr, C., "Nonlinear shape statistics in Mumford-Shah based segmentation", Proc. European conference on computer vision, Copenhagen, 2351, 93-108 (2002).

Cremers, D., Osher, S., Soatto, S., "Kernel density estimation and intrinsic alignment for knowledge-driven segmentation: teaching level sets to walk", Pattern recognition, 3175, 36-44 (2004).

Cremers, D., Sochen, N., Schnorr, C, "Towards recognition-based variation segmentation using shape prior and dynamic labeling", Proc European conference on computer vision, 2685, 388-400 (2003).

De Santis, G., Mortier, P., De Beule, M., Segers, P., Verdonck, P., Verhegge, B., "Patient-specific computational fluid dynamics: structured mesh generation from coronary angiography", Medical \& Biological Engineering \& Computing, 48, 371-380 (2010).

Dutt, V., GrennLeaf., "Ultrasound echo envelope analysis using a homodyned k-distribution signal model", Ultrasound imaging, 16, 365-387 (1994).

Gariepy, J., Massonneau, M., Levenson, J., Heudes, D., Simon, A., "Evidence for in vivo carotid and femoral wall thickness in human hypertension", Journal of Hypertension, 22, 111-118 (1993).

Golemati, S., Stoitsis, J., Sifakis, E. G., Balkizas, T., Nikita, K. S., "Using the Hough transform to segment ultrasound images of longitudinal and transverse sections of the carotid artery", Ultrasound in Medical \& Biological Engineering \& Computing, 33, 1918-1932 (2007).

Guo, Yi, Wang, Yuanyuan, "Automatic endocardium extraction for echocardiogram", 4th Internation Conference on Biomedical Engineering and Informatics (BMEI), Shangai, China, 155-159 (2011).

Gustavsson, T., Liang, Q., Wendelhag, I., Wikstrand, J., "A dynamic programming procedure for automated ultrasonic of the carotid artery", Computers in cardiology, 297-300 (1994).

Haneline, M. T., Croft, A. C., Frishberg, B. M., "Association of internal carotid artery dissection and chiropractic manipulation", The Neurologist, 9, 35-44 (2003).

Hanbay, D., "An expert system based on least square support vector machines for diagnosis of the valvular heart disease", Expert system with Applications, 36, 4232-4238 (2009).

Izquierdo-Zaragoza, J. L., Bastida-Jumilla, M. C., Verdú-Monedero, R., "Segmentation of the carotid artery in ultrasound images using frequency-designed b-spline active contour", Acoustics, Speech and Signal Processing (ICASSP), Prague, 713-716 (2011).

Jegelevicius, D., Lukosevicius, A., "Ultrasonic measurements of human carotid artery wall intima-media thickness", ULTRAGARSAS, 43(2), 43-47 (2002).

Jiaoying, J., Mingyue, D., Yang, X., "Automatic detection of the intima-layer in ultrasound common carotid artery image based on active contour model", Intelligent Computation and Bio-Medical Instrumentation (ICBMI), International Conference on Wuhan, Hubei, 105-108 (2011). 
Kozich, R. J., "Detecting interfaces on ultrasound images of the carotid artery by dynamic programming", SPIE Medical Imaging, 2666, 233-241 (1996).

Latifoglu, F., Sahan, S., Kara, S., Günes, S., "Diagnosis of atherosclerosis from carotid artery Doppler signals as a real-world medical application of artificial immune systems", Experts Systems with Applications, 33, 786-793 (2007).

Loizou, C.P., Pattichis, C. S., Pantziaris, M., "An integrated system for the segmentation of atherosclerosis carotid plaque", IEEE Transactions on information technology in biomedicine, 11, 661-667 (2007).

Ma, Z., Jorge, R. N., Mascarenhas, T., Tavares, J. M. R. S., "A Review of Algorithms for Medical Image Segmentation and their Applications to the Female Pelvic Cavity", Computer Methods in Biomechanics and Biomedical Engineering, 13, 235-246 (2010).

Ma, Z., Jorge, R. N., Mascarenhas, T., Tavares, J. M. R. S., "Novel Approach to Segment the Inner and Outer Boundaries of the Bladdes Wall in T2-weighted Magnetic Resonance Images", Annals of Biomedical Engineering, 39, 2287-2297 (2011).

Ma, Z., Jorge, R. N., Mascarenhas, T., Tavares, J. M. R. S. "Segmentation of Female Pelvic Cavity in Axial T2-weighted MR Images towards the 3D reconstruction", The International Journal for Numerical Methods in Biomedical Engineering, 28, 714-726 (2012).

Matsakou, A. I., Golemati, S., Stoitsis, J. S., "Automated detection of the carotid artery wall in longitudinal Bmode images using active contours initialized by the Hough transform", 33rd annual international conference of the IEEE, EMBS, Boston, Massachusetts, USA, August 30-September 3 (2011).

Meiburguer, K. M., Molinari, F., Acharya, U. R., Saba, L., Rodrigues, P., Liboni, W., Nicholaides, A., Suri, J. S., "Automated carotid artery intima-layer regional segmentation", Physics in medicine and biology, 56, 4073-4090 (2011).

Milner, J. S., Moore, J. A., Rutt, B. K., Steinman, D. A., "Hemodynamics of human carotid artery bifurcation: computational studies reconstructed from magnetic resonance imaging of normal subjects", Journal of vascular surgery, 27, 143-156 (1998).

Molinari, F., Liboni, W., Giustetto, P., Badalamenti, S., "Automated computer-based tracings (ACT) in longitudinal images using different scanners", Journal of mechanics in medicine and biology, 9, 1-25 (2009).

Molinari, F., Liboni, W., Giustetto, P., Suri, J. S., "Accurate and automatic carotid plaque characterization in contrast enhanced 2D ultrasound images", Proceedings of the 29th annual international conference of the IEEE, EMBS, Lyon, France (2007).

Molinari, F., Zeng, G., Suri, J. S., "An integrated approach to computer-based automated tracing and its validation for 200 common carotid artery wall ultrasound images", Journal of Ultrasound in Medicine., 29, 399-418 (2010).

Molthen, R. C., Narayanan, V. M., Shankar, P. M., Reid, J. M., Genis, V., Vergara-Domínguez, L., "Ultrasound echo evaluation by k-distribution", Ultrasonics Symposium IEEE, 957-960 (1993).

Nambi, V., Chambless, L., He, M., Folsom, A. R., Mosley, T., Boerwinkle, E., Ballantyne, C. M., "Common carotid artery intima-media thickness is as good as carotid intima-media thickness of all carotid artery segments in improving prediction of coronary heart disease risk in atherosclerotic risk in communities (ARIC) study", European Heart Journal, 33, 183-190; 
Noble, J. A., Boukerrouni, D., (2006). Ultrasound image segmentation: a survey. IEEE Transaction in medical imaging, 25, 987-1010 (2012).

Osher, S., Fedwik, R., "Level set methods and dynamic implicit surfaces", Springer Verlag, New York, 153 (2003).

Perona, P., Malik, J., "Scale-space and edge detection using anisotropic diffusion", IEEE transaction on pattern analysis and machine intelligence, 12, 1-30 (1990).

Petroudi, S., Loizou, C., Pantziaris, M., Pattichis, M., Pattichis, C. S., "A fully automated method using active contours for the evaluation of the intima-media thickness in carotid US images", 33rd annual international conference of the IEEE, EMBS, Boston, Massachusetts, USA, 8053-8057 (2011).

Petroudi, S., Loizou, C. P., Pattichis, C. S., "Atherosclerosis carotid wall segmentation in ultrasound images using Markov random fields", Information Technology and Applications in Biomedicine (ITAB), 10th IEEE International Conference, Corfu, Greece, 1-5 (2010).

Poonguzhali, S., Ravindran, G., "A complete automatic region growing method for segmentation of masses on ultrasound images", Research Publishing Services, 88-92 (2006).

Rocha, R., Campilho, A., Silva, J., "Segmentation of ultrasonic images of the carotid", International Conference on Image Analysis and Recognition, Toronto, Canada, 3656, 949-957 (2005).

Rocha, R., Campilho, A., Silva, J., Azevedo, E., Santos, R., "Segmentation of the carotid intima-media region in B-mode ultrasound images". Image and Vision Computing, 28, 614-625 (2010).

Rocha, R., Campilho, A., Silva, J., Azevedo, E., Santos, R., "Segmentation of ultrasound images of the carotid using RANSAC and cubic splines", Computer Methods and Programs in Biomedicine, 101, 94-106 (2011).

Rossi, A. C., Brands, P. J., Hoeks, A. P. G., "Automatic recognition of the common carotid artery in longitudinal ultrasound B-mode scans", Medical image analysis, 12, 653-665 (2008).

Sanderse, M., Marquering, H. A., Hendriks, E. A., van der Lugt, A., Reiber, J. H. C., "Automatic initialization algorithm for carotid artery segmentation in CTA images", Medical Image Computing and Computer Assisted Intervention, 3750, 846-853 (2005).

Sarti, A., Corsi, C., Mazzini, E., Lamberti, C., "Maximum likehood segmentation of ultrasound images with Rayleigh distribution", Computers in cardiology, 52, 947-960 (2005).

Schmidt-Trucksass, A., Cheng, D., Sandrock, M., Schulte-Monting, J., Rauramaa, R., Huonker, M., Burkhardt, H., "Computerized analyzing system using the active contour in ultrasound measurement of carotid artery intima-media thickness", Clinical Physiology, 21, 561-569 (2001).

Selzer, R. H., Hodis, H. N., Kwong-Fu, H., Mack, W. J., Lee, P. L., Liu, C. R., Liu, C. H., "Evaluation of computerized edge tracking for quantifying intima-media thickness of the common carotid artery from Bmode ultrasound images", Atherosclerosis, 111, 1-11 (1994).

Serna-Morales, A. F., Prieto-Ortiz, F., Bayro-Corrochano, E., "Acquisition of three-dimensional information of brain structures using endoneurosonography", Expert Systems with Applications, 39, 1656-1670 (2012). 
Sommer, G., Holzapfel, G. A., "3D constitutive modeling of the biaxial mechanical response of intect and layer-dissected human carotid arteries", Journal of the Mechanical Behavior of Biomedical Materials, 5, 116128 (2012).

Stein, J. H., "Use of Carotid Ultrasound to identify subclinical vascular disease and evaluate cardiovascular disease risk: a consensus statement from the American Society of Echocardiography Carotid Intima-Media Thickness Task Force endorsed by the society for vascular medicine", American Society of Echocardiography, 21, 93-111 (2008).

Thijssen, J. M., "Ultrasonic speckle formation and processing applied to tissue characterization", Pattern recognition, 24, 659-675 (2003).

Thwin, S. S., Soe, M. M., Myint, M., Lwin, S., "Variation of the origin and branches of the external carotid artery in human cadaver", Singapore Medical Journal, 51, 40-42 (2010).

Touboul, P. J., Prati, P., Sxarabin, P. Y., Adrai, V., Thibout, E., Ducimetiere, P., "Use of monitoring software to improve the measurement of carotid wall thickness by B-mode imaging", Journal of Hypertension, 10, 3741 (1992).

Unal, G., Bucher, S., Carlier, S., Slabaugh, G., Fang, T., Tanaka, K., "Shape-driven segmentation of the arterial wall in intravascular ultrasound images", IEEE Transaction on information technology in biomedicine, 12(3), 50-57 (2008).

Urquiza, S. A., Blanco, P. J., Vénere, M. J., Feijóo, R. A., "Multidimensional modelling for the carotid artery blood flow", Computer Methods in Applied Mechanics and Engineering, 195, 4002-4017 (2006).

Vukadinovic, D., van Walsum, T., Manniesing, R., Rozie, S., Hameeteman, R., de Weert, T. T., Lugt, A., Niessen, W. J., "Segmentation of the outer wall of the common carotid artery in CTA", IEEE Transactions on Medical Imaging, 9, 65-76 (2010).

Wan, J., Ruan, Q., Li, W., "Using two methods for recognition common carotid artery of B-mode longitudinal ultrasound image", International Conference on Space Optics Proceedings, IEEE 10th International Conference on Beijing, China, 1-4 (2010).

Wagner, R. F., Smith, S. W., Sandrik, J. M., Lopez, H., "Statistical of speckle in ultrasound B-scans", IEEE Transactions, 30(3), 156-163 (1983).

Yan, S., Yuan, J., Hou, C., "Segmentation of Medical Ulstrasound Images Based on Level Set Method with Edge Representing Mask", 3rd International Conference on Advanced Computer Theory and Engineering, 2, 85-88 (2010).

Yang, X., Ding, M., Lou, L., Yuchi, M., Qiu, W., Sun, Y., "Common carotid artery lumen segmentation in Bmode ultrasound transverse view images", International Journal of Image, Graphics and Signal Processing, 5, 15-21 (2011).

Younis, H. F., Kaazempur-Mofrad, M. R., Chan, R. C., Isase, A. G., Hinton, A. H., Kin, L. A., Kamm, R. D., "Hemodynamics and wall mechanics in human carotid bifurcation and its consequences for atherogenesis: investigation of inter-individual variation", Biomechanics and Modeling in Mechanobiology, 3, 17-32 (2004). 